09. 10. - 11. 10. 2008

Hannover

Deutschland

10. 10. - 11. 10. 2008

Tübingen

Deutschland

10. 10. - 11. 10. 2008

Tübingen

Deutschland

Tübingen

Deutschland

…............

11. 10. - 12. 10. 2008

Bremen

Deutschland

1.....

Alpbach/Tirol

Österreich

16. 10. 19.10 .2008

Graz

Österreich

\section{10. 2008}

Tübingen

Deutschland

07.11 .08 .11

Tübingen

Deutschland

07. 11. - 08. 11. 2008

Tübingen

Deutschland

11. 2008

Bad Lippspringe

Deutschland

Tübingen

Deutschland
Verhaltenstherapie 2008;18:204-205
Wider das Stigma - ADHS, Tics und Zwänge im Spiegel von Gesellschaft und Forschung

Trauer und Verlust (C7)

Curriculum forensische Kinder- und Jugendpsychiatrie (FKJ-3) Zivilrecht I

Aufbaukurs für Supervisorinnen und Supervisoren der WiT-Supervisionsausbildung (Sup)

\section{Kinderverhaltenstherapietage}

Kongress Essstörungen 2008 - Eating Disorders 2008

16. Internationale Wissenschaftliche Tagung - 16th International

Conference on Eating Disorders

10. Wissenschaftliche Tagung der AVM-Ö

Thema: «Neurobiologie und Verhaltenstherapie»

Hypnose bei ADHS bei Erwachsenen (C2b)

Grundausbildung Hypnose in der Psychotherapie (B6)

Nutzung von Trancephänomenen II

Curriculum Tabakabhängigkeit und Entwöhnung (PE 2)

Hypnotherapie - Traditionelle HeilKunst heute

Adipositas / Übergewicht: Ein hypnotherapeutisches Gruppenprogramm zur Gewichtsreduktion (C8)
Auskunft:

Kongressbüro Rieke Onken, 49082 Osnabrück

Tel. +49 0541 6002-494, Fax -2493

info@tagung-mhh.de, www.tagung-mhh.de

Auskunft:

WiT - WissensTransfer, Tübingen

Tel. +49 7071 29-76439, Fax -5101

wit@uni-tuebingen.de, www.wit.uni-tuebingen.de

Auskunft:

WiT - WissensTransfer, Tübingen

Tel. +49 7071 29-76439, Fax -5101

wit@uni-tuebingen.de, www.wit.uni-tuebingen.de

Auskunft:

WiT - WissensTransfer, Tübingen

Tel. +49 7071 29-76439, Fax -5101

wit@uni-tuebingen.de, www.wit.uni-tuebingen.de

Auskunft:

Frau Eva Todisco, Zentrum für Klinische Psychologie

und Kinderambulanz der Universität Bremen

Tel. +49 421 218-7075, Fax -4614

todisco@uni-bremen.de, www.zrf.uni-bremen.de

\section{Auskunft:}

Netzwerk Essstörungen, Innsbruck

info@netzwerk-essstoerungen.at,

www.netzwerk-essstoerungen.at



\section{Auskunft:}

Arbeitsgemeinschaft für Verhaltensmodifikation

(AVM-Ö), A-Salzburg

Tel. +43662 884166

office@verhaltenstherapie-avm.at,

www.verhaltenstherapie-avm.at

Auskunft:

WiT - WissensTransfer, Tübingen

Tel. +49 7071 29-76439, Fax -5101

wit@uni-tuebingen.de, www.wit.uni-tuebingen.de

\section{Auskunft:}

WiT - WissensTransfer, Tübingen

Tel. +49 7071 29-76439, Fax -5101

wit@uni-tuebingen.de, www.wit.uni-tuebingen.de

Auskunft:

WiT - WissensTransfer, Tübingen

Tel. +49 7071 29-76439, Fax -5101

wit@uni-tuebingen.de, www.wit.uni-tuebingen.de

Auskunft:

DGH-Geschäftsstelle

Tel. +49 2541 88076, Fax +49 254170008

DGH-Geschaeftsstelle@t-online.de,

www.hypnose-dgh.de

Auskunft:

WiT - WissensTransfer, Tübingen

Tel. +49 7071 29-76439, Fax -5101

wit@uni-tuebingen.de,www.wit.uni-tuebingen.de

\section{KARGER}

Fax +497614520714

Information@Karger.de

www.karger.com

\section{(c) 2008 S. Karger GmbH, Freiburg}




\section{11. - 22, 11. 2008}

Tübingen

Deutschland

26. 11. 29. 11.2008

Berlin

Deutschland

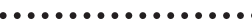

26. 11. - 29. 11.2008

Tübingen

Deutschland

.............

29. 11. - 30. 11. 2008

Tübingen

Deutschland

-

12. 12. - 13. 12. 2008

Tübingen

Deutschland

[.................

19. 12. - 20. 12. 2008

Tübingen

Deutschland

[..................

18.03. - 21.03.2009

Mainz

Deutschland

[...................

21. 05. - 23. 05. 2009

Zürich

Schweiz

•.................

21. 05. - 24. 05.2009

Wien

Österreich

.....................

28. 06. - 02. 07. 2009

Paris

Frankreich

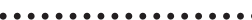

01. 10. - 03. 10. 2009

Leipzig

Deutschland
Curriculum forensische Kinder- und Jugendpsychiatrie (FKJ-4)

Zivilrecht II

\section{DGPPN Kongress}

Leitthema: «Psychiatrie als therapeutische Disziplin»

Weiterbildung zur Supervisorin / zum Supervisor 2. Jahr (PVIII.6)

Weiterbildung im Autogenen Training und in der Hypnose (Imag/Sup) Arbeit mit Imaginationen in leichter Trance (Oberstufe des AT, gestufte

Aktivhypnose) und Entwicklung von Einsatzplänen für AT und Hypnose (kollegiale Supervision)

\section{Die Bearbeitung von Träumen (C9)}

Grundausbildung Hypnose in der Psychotherapie (B7)

Therapeutische Geschichten und Metaphern

60. Arbeitstagung für Psychosomatische Medizin und Ärztliche Psychotherapie (DGPM) und

17. Jahrestagung der Deutschen Gesellschaft für Psychosomatische Medizin und Ärztliche Psychotherapie (DGPM)

6. Workshopkongress für Klinische Psychologie und Psychotherapie

27. Symposium der Fachgruppe Klinische Psychologie und Psychotherapie der DGP

\section{International Congress on ADHD}

9. World Congress of Biological Psychiatry

12. Kongress der Deutschen Gesellschaft für Verhaltensmedizin und Verhaltensmodifikation - DGVM
Auskunft:

WiT - WissensTransfer, Tübingen

Tel. +49 7071 29-76439, Fax -5101

wit@uni-tuebingen.de, www.wit.uni-tuebingen.de

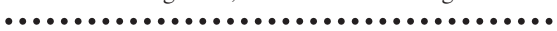

Auskunft:

CPO Hanser Service GmbH, Berlin

Tel. +49 30 300669-0, Fax -50

doppn08@cpo-hanser.de,www.dgppn-kongress.de

•..............................

Auskunft:

WiT - WissensTransfer, Tübingen

Tel. +49 7071 29-76439, Fax -5101

wit@uni-tuebingen.de, www.wit.uni-tuebingen.de

Auskunft:

WiT - WissensTransfer, Tübingen

Tel. +49 7071 29-76439, Fax -5101

wit@uni-tuebingen.de, www.wit.uni-tuebingen.de

\section{Auskunft:}

WiT - WissensTransfer, Tübingen

Tel. +49 7071 29-76439, Fax -5101

wit@uni-tuebingen.de, www.wit.uni-tuebingen.de

Auskunft:

WiT - WissensTransfer, Tübingen

Tel. +49 7071 29-76439, Fax -5101

wit@uni-tuebingen.de, www.wit.uni-tuebingen.de

............................

Auskunft:

Intercongress $\mathrm{GmbH}$, Freiburg

Tel. +49 761 69699-0, Fax -11

info.freiburg@intercongress.de

\section{Auskunft:}

Universität Zürich, Psychologisches Institut,

Klinische Psychologie und Psychotherapie

Tel. +41 44635 73-51, Fax -59

kongress09@psychologie.uzh.ch

\section{Auskunft:}

CPO Hanser Service, Barsbüttel

Tel. +49 40 670-88 20, Fax -32 83

adhd2009@cpo-hanser.de, www.adhd-congress.org

•.........................

Auskunft:

WFSBP Global Headquarters, Barsbüttel

Tel. +49 40670 882-90, Fax -91

info@wfsbp.org,www.wfsbp2009.org

\section{Auskunft:}

www.dgvm-online.de 